\title{
Estudio preliminar de la actividad antioxidante de tres especies del género Ganoderma (Polyporaceae) nativas del estado de Hidalgo, México
}

\section{Preliminary study of antioxidant activity of three species of Ganoderma genus (Polyporaceae) native of Hidalgo State, México}

\author{
Miguel Ángel Islas-Santillán', Araceli Castañeda Ovando², Antonio Álvarez Delgadillo', Ricardo Valenzuela Garza³, \\ Leticia Romero-Bautista', J. Martín Torres-Valencia ${ }^{4}$
}

\begin{abstract}
' Laboratorio de Etnobotánica. Centro de Investigaciones Biológicas. Universidad Autónoma del Estado de Hidalgo. Pachuca, Hidalgo, México.
${ }^{2}$ Laboratorio de Físico-química de Alimentos II. Área Académica de Química. Universidad Autónoma del Estado de Hidalgo. Pachuca, Hidalgo, México. ${ }^{3}$ Instituto Politécnico Nacional, Departamento de Botánica, Escuela Nacional de Ciencias Biológicas. Ciudad de México. ${ }^{4}$ Área Académica de Química, Universidad Autónoma del Estado de Hidalgo. Pachuca, Hidalgo, México.
\end{abstract}

Miguel Ángel Islas Santillán, e-mail: miguel.islass@gmail.com

\section{RESUMEN}

Antecedentes: El metabolismo produce radicales que dañan células y biomoléculas, promoviendo peroxidación lipídica, envejecimiento y enfermedades crónico-degenerativas. El género Ganoderma posee metabolitos con actividad antioxidante, anticancerígena, antitumoral y antiinflamatoria.

Objetivo: Comparar la capacidad antioxidante y el contenido de polifenoles de extractos etanólicos y acuosos (basidiomas, micelio) de tres especies de Ganoderma del estado de Hidalgo.

Métodos: La capacidad antioxidante se evaluó con el método ABTS y los fenoles totales con el método Folin-Ciocalteu. Se analizó la varianza (ANOVA), pruebas de Tukey y análisis discriminante generalizado lineal (AFDG).

Resultados y conclusiones: Existieron diferencias significativas entre extractos acuosos y etanólicos, la mayor actividad antioxidante ocurrió en extractos etanólicos de G. brownii, G. applanatum (GaA-b) y G. curtisii (GcZ-b), y en acuosos de G. brownii, G. applanatum (GaA-m) y G. curtisii (GcA-m); G. brownii (GbT-m) mostró mayor actividad. El análisis AFDG, determinó 77.96\% de varianza en la primera función discriminante por capacidad antioxidante y polifenoles de extractos etanólicos, y $20.07 \%$ en la segunda por correlación de fenoles de ambos extractos y antioxidantes en acuosos. Los resultados indican que la actividad antioxidante se asocia a la presencia de fenoles, polisacáridos y esteroles, variando según la especie, etapa de desarrollo, extracto y lugar de colecta.

Palabras clave: radical libre, ABTS, esteroles, polifenoles/fenoles

\section{ABSTRACT}

Background: Metabolism produce free radicals, which damage cells and biomolecules, causing lipid peroxidation, aging process and chronic degenerative diseases. The genus Ganoderma has metabolites with antibacterial, anti-cancer, anti-tumor, anti-inflammatory and antioxidant activities.

Objective: Compare antioxidant capacity and total polyphenols content of ethanolic and aqueous extracts (basidiomes and mycelium) of three species of Ganoderma from Hidalgo State.

Methods: Antioxidant capacity was evaluated by the ABTS method and total polyphenols content by the Folin-Ciocalteu method. Statistical tests included variance analysis (ANOVA), Tukey test and generalized linear discriminant analysis (AFDG). 
Results and conclusions: Significant differences were observed between aqueous and ethanolic extracts, the highest activity happened in ethanolic extracts of G. brownii, G. applanatum (GaA-b) and G. curtisii (GcZ-b) and aqueous extracts of G. brownii, G.applanatum (GaA-m) and G. curtisii (GcA-m), the most active was G. brownii (GbT-m). The AFDG analysis explained $77.96 \%$ of the variance in first discriminant function by antioxidant capacity and phenols present in ethanolic extracts and $20.07 \%$ in second function correlating phenols of both extracts and antioxidants in aqueous extracts. These results show that antioxidant activity is determined by the presence of phenols, polysaccharides and sterols, varying according to the species, stage of development, extract and collection area.

Key words: free radical, ABTS, sterols, polyphenols/phenols

\section{INTRODUCCIÓN}

Como resultado del metabolismo, algunos organelos celulares como mitocondrias, lisosomas, peroxisomas, membrana nuclear, citoplásmica y retículo endoplásmico originan radicales libres, como las especies reactivas de oxígeno (ROS): superóxido $\left(\mathrm{O}_{2}{ }^{-}\right)$, hidroxilo $\left(\mathrm{OH}^{*}\right)$, peroxilo (R-OO'), alcoxilo $\left(\mathrm{RO}^{*}\right)$ e hidroperoxilo (HOO`) y las especies reactivas de nitrógeno (RNS): óxido nítrico (NO), dióxido de nitrógeno $\left(\mathrm{NO}_{2}\right)$ y peróxido de nitrato (Maldonado et al., 2010). Sin embargo, éstos también pueden generarse por factores externos como contaminación ambiental, exposición a radiación y productos químicos como medicamentos, aditivos alimenticios, pesticidas, herbicidas y tabaco, entre otros.

Estos radicales atacan moléculas como aminoácidos, carbohidratos, lípidos, proteínas y ácidos nucleicos. Para contrarrestar su efecto, las células han desarrollado mecanismos de protección y reparación del daño, como la producción de enzimas antioxidantes como superóxido dismutasa, catalasa, glutatión peroxidasa, glucosa-6-fosfato deshidrogenasa, NADPH-quinona oxidorreductasa y epóxido hidrolasa (Finkel y Holbrook, 2000) y de metabolitos antioxidantes que atrapan y neutralizan los radicales libres; sin embargo, resultan insuficientes para protegerlas completamente (Smina et al., 2011).

Cuando los oxidantes superan la cantidad de antioxidantes ocurre un estrés oxidativo, que provoca daños importantes en biomoléculas y componentes celulares (Halliwell, 1996), lo cual está asociado con enfermedades crónico degenerativas como Alzhaimer, Parkinson, arteriosclerosis, cáncer, artritis, desórdenes neurodegenerativos, enfermedades coronarias y envejecimiento, entre otras (Yoshikawa et al., 2000; Álvarez et al., 2008; Badarinath et al., 2010; Kozarski et al., 2011).

Debido a lo anterior, es importante la búsqueda de productos naturales con propiedades antioxidantes que al incluirse en la dieta diaria contribuyan a mantener la salud, al prevenir y curar enfermedades, siendo interesante el estudio de especies fúngicas que poseen potencial medicinal. Existen varios hongos medicinales entre ellos los del género Ganoderma que tienen agentes antioxidantes como polifenoles, terpenoides, triterpenos y esteroles (Smina et al., 2011), como el ergosterol y sus derivados que reducen la peroxidación lipídica (Kobori et al., 2007) y poseen propiedades antitumorales y anticancerígenas (Wasser y Weis, 1997).

Ganoderma es un género lignícola que crece en ambientes tropicales causando pudrición blanca de duramen y raíz especialmente en latifoliadas y algunas coníferas (Cibrián-Tovar et al., 2007), entre ellas Quercus spp., Ulmus spp., Acer spp., Fraxinus spp., Acacia spp., Populus spp., Macadamia spp., Abies spp, Tsuga sp., Cocus nucifera, Areca catechu, Prunus persica, Pyrus communis, Paullinia cupana, Vitis spp. y Coffea spp. (Sánchez, 1980; Ariffin et al., 2000; Stamets, 2000; Pilotii et al., 2004; Alvarado-Rosales et al., 2007).

Entre los trabajos sobre actividad antioxidante para el género Ganoderma, destacan los de Mau et al. (2005a,b) y Tseng et al. (2008) que evaluaron extractos acuosos y metanólicos, así como compuestos polisacáridos de basidiomas de G. tsugae; Chen et al. (2008) realizaron pruebas con polisacáridos de basidiomas de G. atrum; Saltarelli et al. (2009) cuantificaron la actividad del micelio de G. lucidum; Kalyoncu et al. (2010) con el micelio de 21 especies silvestres incluyendo G. lucidum; Smina et al. (2011) probaron la fracción triterpénica de G. lucidum con diferentes métodos in-vitro e in-vivo; Kozarski et al. (2011) con la fracción polisacárida de cuatro hongos medicinales incluido $G$. lucidum; Ćilerdžić et al. (2014) evaluaron basidiomas cultivados de G. lucidum mientras que Nagaraj et al. (2014) y Acharya et al. (2015) los basidiomas silvestres de G. applanatum.

Singdevsachan et al. (2015) compararon la composición química y actividad de G. applanatum, G. lipsiense, G. chal- 
ceum y G. tsugae. Por otra parte, Tamrakar et al. (2016) estudiaron 62 especies de hongos silvestres de Nepal incluidas cuatro de Ganoderma. Para México destaca el trabajo de Garza et al. (2006) que investigaron la actividad biológica de extractos acuosos de cuatro especies fúngicas del noreste de México incluyendo G. applanatum y el de Huerta et al. (2016) que estimaron la actividad de extractos alcohólicos e hidroalcohólicos de basidiomas silvestres de G. curtisii del estado de Michoacán. Sin embargo, existen pocos estudios sobre la composición química y actividad biológica de especies de Ganoderma nativas de México, que confirmen las propiedades atribuidas a este género.

El objetivo del trabajo fue determinar la capacidad antioxidante y el contenido de polifenoles totales presentes en extractos etanólicos y acuosos de basidiomas y micelio de Ganoderma curtisii, G. applanatum y G. brownii, procedentes de tres localidades del estado de Hidalgo, México.

\section{MATERIALES Y MÉTODOS}

\section{Recolecta de material biológico}

Se recolectaron basidiomas maduros de Ganoderma curtisii, G. applanatum y G. brownii en bosques templados de tres localidades del Estado de Hidalgo (Tabla 1). Los ejemplares fueron identificados siguiendo técnicas convencionales de taxonomía (Largent, 1973; Largent et al., 1977; Gilbertson y Ryvarden, 1986; Ryvarden, 1991) y las claves de identificación para el género propuestas por Ryvarden y Gilbertson (1993), CibrianTovar et al. (2007) y Torres et al. (2015); posteriormente fueron deshidratados a temperatura ambiente en obscuridad. Un espécimen de cada especie se depositó en la colección de hongos "Dr. Gastón Guzmán Huerta” del herbario de la Escuela Nacional de Ciencias Biológicas (ENCB) del Instituto Politécnico Nacional, con los números de registro IslasGA10, IslasGA14, IslasGAB20, IslasGCT36, IslasGCZ28 (Tabla 2).

Tabla 1. Localidades de recolecta de basidiomas de Ganoderma spp.

\begin{tabular}{llccc}
\hline \multicolumn{1}{c}{ Localidad } & \multicolumn{1}{c}{ Municipio } & Vegetación & Altitud (msnm) & Ubicación Geográfica \\
\hline Los Reyes & Acaxochitlán & BQP & 2252 & $20^{\circ} 09^{\prime} 17^{\prime \prime} ; 98^{\circ} 09^{\prime} 52^{\prime \prime}$ \\
Cuatempa & Tlanchinol & BMM & 1474 & $20^{\circ} 59^{\prime} 04^{\prime \prime} ; 98^{\circ} 37^{\prime} 28^{\prime \prime}$ \\
La Mojonera & Zacualtipán & BF & 2012 & $20^{\circ} 37^{\prime} 82^{\prime \prime} ; 98^{\circ} 36^{\prime} 91^{\prime \prime}$ \\
\hline
\end{tabular}

BPQ (bosque de Quercus-Pinus), BMM (bosque mesófilo de montaña), BF (bosque de Fagus).

Tabla 2. Listado de muestras utilizadas en la preparación de extractos

\begin{tabular}{|c|c|c|c|c|}
\hline Especie & Procedencia & Muestra & Clave Herbario/cepario & Clave de muestra \\
\hline \multirow[t]{3}{*}{ G. curtisii } & Acaxochitlán & Basidioma (silvestre) & IslasGA14 & GcA-b \\
\hline & & Basidioma (cultivado) & & GcA-bc \\
\hline & & Micelio (cepa) & UAEHOOI7 & GcA-m \\
\hline G. curtisii & Tlanchinol & Basidioma (silvestre) & IslasGCT36 & GcT-b \\
\hline G. curtisii & Zacualtipán & Basidioma (silvestre) & IslasGCZ28 & GcZ-b \\
\hline \multirow[t]{2}{*}{ G. brownii } & Tlanchinol & Basidioma (silvestre) & IslasGAB2O & GbT-b \\
\hline & & Micelio (cepa) & UAEH0O26 & GbT-m \\
\hline \multirow[t]{2}{*}{ G. applanatum } & Acaxochitlán & Basidioma (silvestre) & IslasGA10 & GaA-b \\
\hline & & Micelio (cepa) & UAEOОЗО & GaA-m \\
\hline
\end{tabular}




\section{Cultivo en laboratorio}

Se realizaron aislamientos somáticos a partir de basidiomas silvestres (IslasGA14, IslasGAB20, IslasGA10), en cajas Petri con medio malta-peptona-levadura-agar (MYPA) (Shoji, 1999; Postemsky et al., 2006) suplementado con benomil (3 ppm) y aureomicina (10 ppm) para prevenir crecimiento de contaminantes (Stalpers, 1978). Las cepas obtenidas se registraron con las claves UAEH0018 (G. curtisii), UAE0019 (G. applanatum) y UAEH0020 (G. brownii) (Tabla 2). Posteriormente se propagaron en medio líquido malta-levadura-peptona (MYP) a $25^{\circ} \mathrm{C}$ durante 15 días, el micelio obtenido se lavó con agua y se secó a temperatura ambiente en obscuridad. La cepa UAEH0018 (G. curtisii) se cultivó en aserrín de encino suplementado con salvado, en bolsas de $2 \mathrm{~kg}$ que se incubaron a $25^{\circ} \mathrm{C}$ durante 1 mes. Una vez invadidas se inició el riego y la fructificación, transcurridos 3 meses se recolectaron los basidiomas y se deshidrataron a temperatura ambiente en oscuridad.

\section{Preparación de extractos}

Todas las muestras deshidratadas se molieron manualmente. Para los extractos etanólicos se colocaron $2 \mathrm{~g}$ de muestra en 100 $\mathrm{mL}$ de etanol durante $24 \mathrm{~h}$ con agitación magnética en oscuridad, se filtró y aforó a $100 \mathrm{~mL}$ con etanol. En los extractos acuosos se preparó una infusión con 2 g de muestra en 100 mL de agua destilada en ebullición, de igual forma se filtró y aforó a $100 \mathrm{~mL}$.

\section{Pruebas antioxidantes}

Ensayo ABTS. La capacidad antioxidante se evaluó con el método de ABTS (Re et al., 1999), el radical ABTS•+ se obtiene mediante la reacción de una solución stock de ABTS ácido 2,2'-azino-bis(3-etilbenzotiazolin-6-ácido sulfónico) (Sigma) 7 $\mathrm{mM}(19.4 \mathrm{mg})$ con persulfato potásico $2.45 \mathrm{mM}$ (3.3. $\mathrm{mg})$ en 5 $\mathrm{mL}$ de agua desionizada, la solución se almacenó en oscuridad a temperatura ambiente por $16 \mathrm{~h}$ antes de su uso. Posteriormente se diluyó con etanol hasta obtener una absorbancia de 0.60 $( \pm 0.1)$ a una longitud de máxima absorción de $734 \mathrm{~nm}$. La curva de calibración se realizó con una solución estándar de Trolox (6-hidroxi-2,5,7,8-tetrametilcromo-2-ácido carboxílico) $1 \mathrm{mM}$ (2.5 mg en $10 \mathrm{~mL}$ de etanol) a partir de la cual se obtuvieron concentraciones en un intervalo de $10-50 \mu \mathrm{M}$. Los extractos a evaluar se diluyeron con etanol en una proporción 1:10 y 1:5, se colocaron $100 \mu \mathrm{L}$ de cada muestra en una celda espectrofotométrica y se adicionaron $1000 \mu \mathrm{L}$ de $\mathrm{ABTS}^{\bullet+}$, las lecturas se realizaron por triplicado en un espectrofotómetro marca Jenway 6405UV/Vis a una absorbancia de $734 \mathrm{~nm}$ durante $10 \mathrm{~min}$, los resultados se expresaron en mg equivalentes Trolox por gramo de muestra (mg Trolox/g).

Contenido de fenoles totales. La cuantificación de fenoles totales se realizó con el método de Folin Ciocalteu (F-C) (Singleton, et al., 1999), utilizando como buffer $\mathrm{Na}_{2} \mathrm{CO}_{3}$ al 4\%, la curva de calibración se hizo utilizando como estándar una solución de ácido gálico $2.5 \mu \mathrm{M}$ (10 mg en $10 \mathrm{~mL}$ de agua destilada) a partir de la cual se prepararon concentraciones en un intervalo de 20-100 $\mu \mathrm{M}$. En un matraz se colocó $1 \mathrm{~mL}$ de muestra, $1 \mathrm{~mL}$ de reactivo de Folin (Sigma), $4 \mathrm{~mL}$ de solución de $\mathrm{Na}_{2} \mathrm{CO}_{3}$ y se aforó a $10 \mathrm{~mL}$ con agua destilada, se calentó a $50{ }^{\circ} \mathrm{C}$ en baño María por 10 min y se midió la absorbancia por triplicado a 765 $\mathrm{nm}$. Las muestras se analizaron de igual forma sustituyendo el patrón de ácido gálico por $1 \mathrm{~mL}$ del extracto a evaluar. Los resultados promedio de las lecturas se expresaron en mg equivalentes de ácido gálico por gramo de muestra (mg EAG/g).

Los datos se estandarizaron mediante la siguiente fórmula:

$$
\mathrm{CR}=\frac{\left(\frac{\mathrm{A}-\mathrm{b}}{\mathrm{m}}\right) * \mathrm{PM}}{\mathrm{C}}=\frac{\mathrm{mg} \text { de referencia }}{\mathrm{g} \text { de muestra }}
$$

En donde $\mathrm{CR}=$ concentración del referente, $\mathrm{A}=$ absorbancia, $\mathrm{m}=$ pendiente de la curva patrón, $\mathrm{b}=$ ordenada al origen de la curva patrón, $\mathrm{PM}=$ peso molecular del compuesto de referencia $(\mathrm{g} / \mathrm{mol})$ y $\mathrm{C}=$ concentración de la muestra $(\mathrm{g} / \mathrm{mL})$.

Los datos resultantes se evaluaron con un análisis de varianza (ANOVA) y se compararon las medias con una prueba de Tukey $(\mathrm{p}<0.05)$. Así mismo, se realizó un análisis discriminante generalizado lineal (AFDG), con los datos de actividad antioxidante expresados en mg Trolox/g de muestra y con la concentración de fenoles totales en mg EAG/g de muestra.

\section{Resultados}

\section{Actividad antioxidante}

Para los extractos etanólicos se formaron tres grupos con la prueba de Tukey, la mayor capacidad antioxidante se observó 
en Ganoderma brownii (GbT-b y GbT-m con 68.54 y 67.82 mg Trolox/g respectivamente), G. applanatum (GaA-b con $67.53 \mathrm{mg}$ Trolox/g), G. curtisii (GcZ-b con $66.38 \mathrm{mg}$ Trolox/g) y G. curtisii (GcA-b con $64.94 \mathrm{mg}$ Trolox/g); y la menor en G. curtisii (GcA-bc y GcA-m con 47.82 y $50.41 \mathrm{mg}$ Trolox/g) (Figura 1).

En los extractos acuosos se integraron cuatro grupos con la prueba de Tukey los valores más altos se obtuvieron en basidiomas de G. curtisii (GcA-m con $67.96 \mathrm{mg}$ Trolox/g), G. brownii (GbT-b con $66.81 \mathrm{mg}$ Trolox/g), G. curtisii (GcA-m con 66.387 mg Trolox/g) y G. brownii (GbT-m con $65.24 \mathrm{mg}$ Trolox/g); los valores más bajos se observaron en G. curtisii (GcA-b y GcA-bcm con 56.45 y $59.76 \mathrm{mg}$ Trolox/g) (Figura 1).

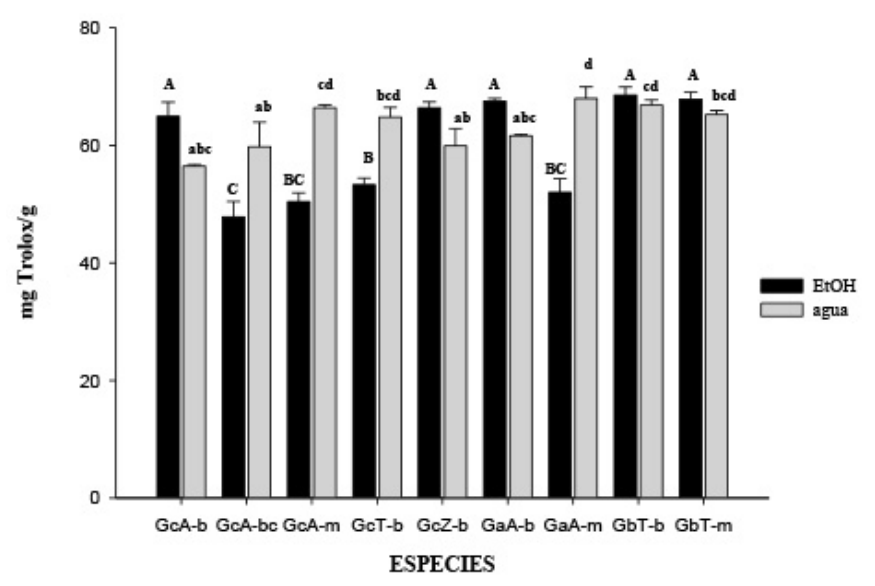

Figura 1. Actividad antioxidante de los extractos crudos de Ganoderma curtisii, G. applanatum y G. brownii, el valor promedio $(\mathrm{n}=3)$ se expresa en mg equivalentes de Trolox por gramo de muestra (mg Trolox/g). Letras diferentes representan diferencias significativas según Tukey $(\alpha=0.05)$, mayúsculas para extractos etanólicos y minúsculas para acuosos.

\section{Cuantificación de fenoles}

La prueba de Tukey permitió diferenciar siete grupos para los extractos etanólicos y nueve para los acuosos (Figura 2). La mayor cantidad de fenoles totales se determinó en extractos etanólicos de G. brownii (GbT-m con $100.33 \mathrm{mg}$ EAG/g) y G. applanatum (GaA-b con $61.36 \mathrm{mg}$ EAG/g) y los valores más bajos en G. curtisii (GcA-m y GcA-b con 13.57 y 19.09 mg
EAG/g, respectivamente). En los extractos acuosos, los valores máximos fueron para G. curtisii (GcA-bc con $146.64 \mathrm{mg}$ EAG/g), G. brownii (GbT-b con 104.37 mg EAG/g) y G. curtisii (GcA-m con 96.65 mg EAG/g) y los valores más bajos para G. applanatum (GaA-m), G. curtisii (GcT-b) y G. applanatum (GaA-b con 47.76, 55.11 y $58.79 \mathrm{mg}$ EAG/g respectivamente) (Figura 2).

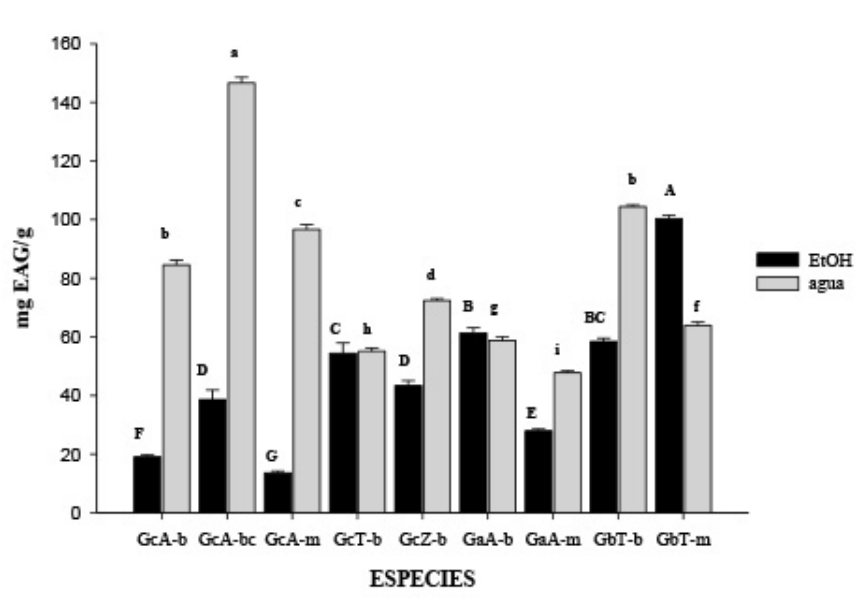

Figura 2. Cuantificación de fenoles totales en los extractos crudos de Ganoderma curtisii, G. applanatum y G. brownii, expresados en miligramos equivalentes de acido gálico por gramo de muestra (mg EAG/g). Letras diferentes representan diferencias significativas según Tukey $(\alpha=0.05)$, mayúsculas para extractos etanólicos y minúsculas para acuosos.

Para comprobar la relación entre el contenido de polifenoles y la actividad antioxidante, se realizó una prueba de correlación para los extractos etanólicos y acuosos por separado, observándose correlación positiva en los extractos etanólicos $\left(\mathrm{R}^{2}=0.280\right.$ y $\mathrm{P}=0.004)$; sin embargo para los extractos acuosos no hubo correlación $\left(\mathrm{R}^{2}=0.072\right.$ y $\left.\mathrm{P}=0.173\right)$.

En el análisis de ANOVA anidado se obtuvo un efecto significativo $(\mathrm{P}=<0.0001)$ de la localidad (solvente) y especie (localidad-solvente) sobre el contenido de polifenoles totales y la capacidad antioxidante; sin embargo, no se observó un efecto significativo por sí solo del solvente utilizado para obtener los extractos en las variables, sino en la interacción (Tabla 3). 
Tabla 3. Análisis de ANOVA anidada de los extractos etanólicos y acusosos de Ganoderma spp.

\begin{tabular}{lcccc}
\hline FACTOR & gl & SC & F & P \\
\hline localidad \{solvente\} & 4 & 487.71 & 36.48 & $<0.0001$ \\
especie \{localidad, solvente\} & 12 & 1696.42 & 42.3 & $<0.0001$ \\
solvente & 1 & 3.24 & 0.97 & 0.33 \\
\hline
\end{tabular}

En el análisis discriminante generalizado AFDG de la actividad antioxidante y de fenoles totales, se obtuvo que el $77.96 \%$ de la varianza se explica por el contenido de fenoles de los extractos etanólicos mg EAG/g (r=-0.033) y por los mg Trolox/g de los extractos etanólicos $(\mathrm{r}=-0.325)$ en la primera función discriminante (FD1). Por otra parte, para la segunda función discriminante (FD2) las variables que más se correlacionaron fueron fenoles de los extractos etanólicos mg EAG/g ( $\mathrm{r}=0.558)$, fenoles y antioxidantes de los extractos acuosos ( $r=0.051$ y $r=-0.193$, respectivamente) con lo cual se explicó el $20.07 \%$ de la variación total (Tabla 4).
Tabla 4. Análisis de función discriminante que muestra la correlación de las variables con las funciones discriminantes, valores propios, significancia $(\mathrm{P})$ y porcentaje de varianza $(\%)$

\begin{tabular}{lcc}
\hline Variable & FD 1 & FD 2 \\
\hline Fenoles acuoso & 0.867 & 0.151 \\
Fenoles etanólico & -0.033 & 0.559 \\
Antioxidante etanólico & -0.326 & 0.014 \\
Antioxidante acuoso & 0.027 & -0.194 \\
Valores propios & 1058.54 & 272.56 \\
$P$ & $<0.0001$ & $<0.0001$ \\
\% Varianza & 77.96 & 20.07 \\
\hline
\end{tabular}

En este análisis se establecieron cinco grupos, en el primero se encuentra G. brownii (GbT-m) con el mayor contenido de polifenoles etanólicos; el segundo grupo integrado por G. curtisii (GcT-b y GcZ-b) y G. applanatum (GaA-b) que exhiben una capacidad antioxidante intermedia para los extractos acuosos (54 mg Trolox/g); en el tercer grupo se ubica G. brownii (GbT-b)

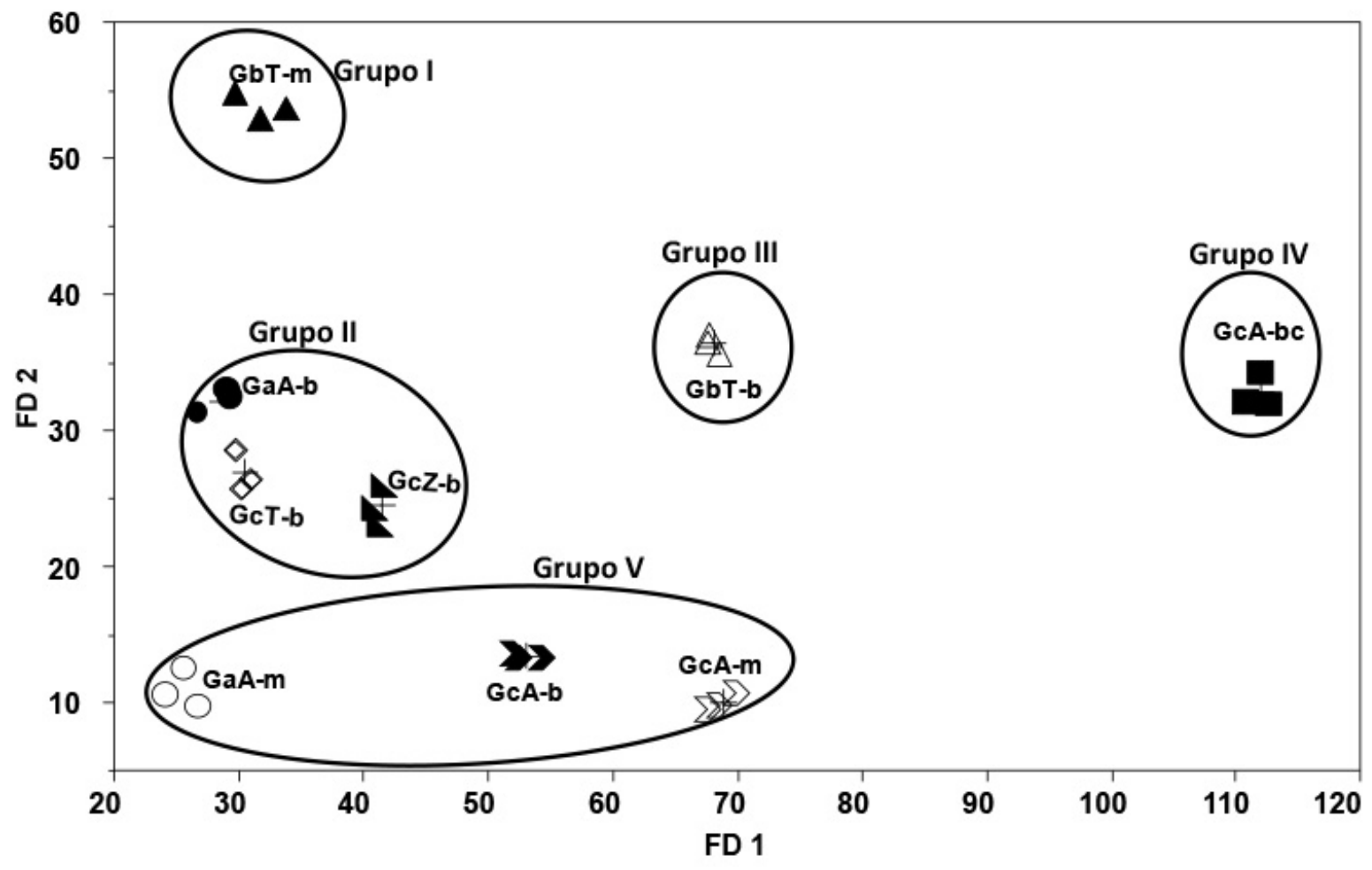

Figura 3. Análisis discriminante generalizado lineal (AFDG) de la actividad antioxidante de los extractos de Ganoderma spp. Grupo 1: G. brownii micelio(GbT-m), grupo 2: G. curtisii basidioma (GcT-b y GcZ-b) y G. applanatum basidioma(GaA-b); grupo 3: G. brownii basidioma (GbT-b); grupo 4: G. curtisii basidioma cultivado(GcA-bc) y grupo 5: G. curtisii micelio (GcA-m) y basidioma (GcA-b) y G. applanatum micelio (GaA-m). 
con una proporción intermedia en polifenoles y capacidad antioxidante de la fracción etanólica pero una mayor cantidad de polifenoles en la fracción acuosa (104.37 mg EAG/g); en el cuarto grupo con una menor actividad se encuentra $G$ curtisii (GcA-bc) con menor cantidad de polifenoles y capacidad antioxidante baja en los extractos etanólicos, sin embargo, contiene la mayor cantidad de polifenoles en los extractos acuosos (146.65 mg EAG/g); y el quinto grupo conformado por G. curtisii (GcA-b, GcA-m) y G. applanatum (GaA-m) con los valores de polifenoles más bajos para los extractos etanólicos (Figura 3).

\section{DISCUSIÓN}

Se analizaron nueve muestras correspondientes a tres especies silvestres en dos etapas de desarrollo: cinco basidiomas silvestres y uno cultivado, además de tres en fase miceliar, todas originarias de tres localidades del estado de Hidalgo, México. El propósito de comparar dos disolventes de alta polaridad (agua y etanol) fue demostrar si existía un efecto en la capacidad antioxidante y contenido de polifenoles de las muestras, debido a que el consumo tradicional de basidiomas del género Ganoderma es principalmente mediante infusiones y tinturas.

Los extractos acuosos de G. curtisii (GcT-b, GcA-m y GcA-bc) y G. applanatum (GaA-m) mostraron mayor actividad antioxidante que los extractos etanólicos, lo que corresponde con lo reportado para G. lucidum (Kalyoncu et al., 2010) y G. tusgae (Mau et al., 2005 a,b). Sin embargo, difiere en G. brownii (GbT-by Gb), G. curtisii (GcZ-b y GcA-b) y G. applanatum (GaA-b), que mostraron una mayor actividad en los extractos etanólicos en relación con los acuosos, lo que es similar a lo reportado para G. applanatum, G. chalceum y G. tsugae (Singdevsachan, et al., 2015).

El contenido de fenoles totales en las muestras analizadas de Ganoderma, fue mayor a lo reportado para algunos hongos comestibles en extractos hidroalcohólicos de Agaricus bisporus (19.97 mg EAG/g), Pleurotus eryngii (12.74 mg EAG/g), Lentinula edodes (10 mg EAG/g) y Pleurotus ostreatus (16.05 mg EAG /g) (Penzete et al., 2012) y en extractos metanólicos de Agaricus bisporus variedad blanca y café $(23.34$ y $37.33 \mathrm{mg}$ EAG/g), Pleurotus ostreatus (12.54 mg EAG/g), Pleurotus eryngii (7.14 mg EAG/g) y Lentinula edodes (8.84 mg EAG/g) (Reis et al., 2012). La cantidad de polifenoles de los extractos etanó- licos de basidiomas de G. curtisii cultivado (GcA-bc) y basidiomas silvestres de G. curtisii (GcZ-b y GcT-b) corresponden con lo obtenido para extractos hidroalcohólico y etanólico de $G$. curtisii (35.63 mg EAG/g y $49.1 \mathrm{mg}$ EAG/g) (Huerta et al., 2016) y para extractos etanólicos de G. lucidum (33-52 mg EAG/g) (Ćilerdžić et al., 2014). Para G. applanatum se observó que el contenido de fenoles en los extractos etanólico y acuoso (61.36 y $58.79 \mathrm{mg}$ EAG/g) es análogo a lo reportado en trabajos similares entre 47 y 71 mg (Kozarski et al., 2011; Nagaraj et al., 2014).

Al comparar el extracto acuoso del micelio de G. curtisii, se obtuvo que la concentración de $96.65 \mathrm{mg}$ EAG/g, fue mayor a lo encontrado para micelio de G. tsugae (41.3 mg EAG/g) (Mau et al., 2005b) a diferencia del extracto etanólico que tuvo una concentración de $13.57 \mathrm{mg}$ EAG/g, menor a lo reportado para el extracto metanólico de G. tsugae (35.6 mg EAG/g) (Mau et al., 2005a) y para extractos hidroalcohólicos de dos cepas de G. Iucidum (Italia y China) con valores de 27.9 y $16.5 \mathrm{mg}$ equivalentes de ácido cafeico/g, respectivamente (Saltarelli et al., 2009).

La actividad antioxidante se ha relacionado con la presencia de compuestos de bajo peso molecular (fenoles) responsables de capturar radicales libres (Gursoy et al., 2009). Sin embargo, también contribuyen los triterpenos (Smina et al., 2011) y polisacáridos de alto peso molecular, como se demostró en pruebas con G. tsugae (Tseng et al., 2008)] y G. curtisii (Saltarelli et al., 2009), estos compuestos además poseen propiedades reguladoras del sistema inmune, anti-radiación, anti-coagulantes, anticáncer, anti-HIV e hipoglicémicas (Lee et al., 2002; Yoon et al., 2003; Yang et al., 2005). De igual forma, se conoce que los micoesteroles como el ergosterol y sus derivados, contribuyen a inhibir la peroxidación lipídica (Kobori et al., 2007).

Las diferencias en las muestras analizadas indican que la concentración de metabolitos secundarios (polisacáridos, triterpenos, fenoles y esteroles) puede variar dependiendo de la especie y etapa de desarrollo. Se observó que los basidiomas maduros de las especies no laqueadas (G. brownii y G. applanatum) presentaron mayor actividad antioxidante y contenido de compuestos fenólicos que las laqueadas (G. curtisii). La variación existente entre las dos etapas de desarrollo pudiera deberse a que el micelio (fase inmadura) se conforma de hifas generativas y esqueléticas que producen enzimas lignolíticas como oxidasas 
y peroxidadas (lacasas, lignino peroxidasa y manganeso peroxidasa) para colonizar el sustrato (Arboleda y Mejía, 2010), mientras que los basidiomas al ser estructuras especializadas en donde se forman las esporas tienen mayor cantidad de triterpenos y polisacáridos. Así mismo, se observó que el disolvente utilizado y la temperatura de extracción influyeron en la actividad antioxidante y el contenido de polifenoles dependiendo de la especie y etapa desarrollo. De igual forma, los factores bióticos y abióticos de las localidades de recolecta pudieran influir en la composición química de las muestras, sin embargo no se analizaron a profundidad, por lo que sería conveniente realizar un estudio complementario. En este estudio preliminar se destaca el potencial antioxidante que tienen las cepas nativas de Ganoderma spp. del estado de Hidalgo con resultados promisorios que motivan la realización de investigaciones futuras enfocadas a la biotecnología y biomedicina.

\section{AGRADECIMIENTOS}

Se agradece al Consejo Nacional de Ciencia y Tecnología de México (CONACYT) la beca otorgada a Miguel Ángel Islas Santillán para la realización del Doctorado en Ciencias en Biodiversidad y Conservación, y por el financiamiento parcial (proyecto CB-2014 No. 238206).

\section{LITERATURA CITADA}

Acharya, K., P. Yonzone, M. Rai, R. Acharya, 2005. Antioxidant and nitric oxide synthase activation properties of Ganoderma applanatum. Indian Journal of Experimental Biology 43: 926-929.

Álvarez, E., O. Jiménez, C. Posada, B. Rojano, J. Gil, C. García, 2008. Actividad antioxidante y contenido fenólico de los extractos provenientes de las bayas de dos especies del genero Vismia (Guttiferae). Vitae 15: 165-172.

Alvarado-Rosales, D., L. de L. Saavedra-Romero, A. Almaraz-Sánchez, B. Tlapal-Bolaños, O. Trejo-Ramírez, J.M. Davidson, J.T. Kliejunas, S. Oak, J.G. O’Brien, F. Orozco-Torres, D. Quiroz-Reygadas, 2007. Agentes asociados y su papel en la declinación y muerte de encinos en México. Polibotánica 23: 1-21.

Arboleda, C., A.I. Mejía, 2010. Inducción de la actividad de lacasa en Ganoderma sp. y actividad antioxidante de su biomasa. Revista Cubana de Farmacia 44: 519-532.

Ariffin, D., A.S. Idris, G. Singh, 2000. Status of Ganoderma in oil palm. In: Flood, J., P.D. Bridge, M. Holdernes (eds.), Ganoderma diseases of perennial crops. CABI Publishing. Londres. Pp. 49-68.

Badarinath, A., K.M. Rao, C.M.S. Chetty, S. Ramkanth, T. Rajan, K.A. Gnanaprakash, 2010. A review on in-vitro antioxidant methods: comparisons, correlations and considerations. International Journal of Pharm Tech Research 2: 1276-1285.

Chen, Y., X. Ming-Yong, N. Shao-Ping, L. Chang, W. Yuan-Xing, 2008. Purification, composition analysis and antioxidant acti- vity of a polysaccharide from the fruiting bodies of Ganoderma atrum. Food Chemestry 1: 231-241.

Cibrián-Tovar, D., D. Alvarado-Rosales, S.E. García-Díaz (Eds.), 2007. Enfermedades forestales en México. Universidad Autónoma Chapingo. Chapingo, México.

Ćilerdžić, J., J. Vukojević, M. Stajić, T. Stanojković, J. Glamočlija, 2014. Biological activity of Ganoderma lucidum basidiocarps cultivated on alternative and commercial substrate. Journal of Ethnopharmacology 155: 312-319.

Finkel, T., N.J. Holbrook, 2000. Oxidants, oxidative stress and the biology ofageing. Nature 408: 239-247.

Garza, L., X.S. Ramírez, F. Garza, M.C. Salinas, N. Waksman, Y. Alcaráz, O. Torres, 2006. Evaluación de la actividad biológica de extractos acuosos de macromicetos del noreste de México. Ciencias Universidad Autónoma de Nuevo León 9: 164-170.

Gilbertson, R.L., L. Ryvarden, 1986. North American Polypores. Volume 1: Abortiporus - Lindtneria. 433 S., 209 Abb. Oslo Fungiflora A/S.

Gursoy, N., C. Sarikürkçü, M. Cengiz, M.H. Solak, 2009. Antioxidant activities, metal contents, total phenolics and flavonoids of seven Morchella species. Food and Chemical Toxicology 47: 23812388.

Halliwell, B., 1996. Oxidative stress, nutrition and health. Free Radical Research 25: 57-74.

Huerta, I., J. Molina, M.G. Garnica, B. Yahuaca, 2016. Total polyphenols and antioxidant activity of Ganoderma curtisii extracts. Journal of Medicinal Plants Studies 4: 136-141.

Kalyoncu, F., M. Oskay, K. Hüsniye, 2010. Antioxidant activity of the mycelium of 21 wild mushroom species. Mycology 1: 195-199.

Kobori, M., M. Yoshida, M. Ohnishi-Kameyama, H. Shinmoto, 2007. Ergosterol peroxide from an edible mushroom supresses inflamatory responses in RAW26.7 macrofages and growth of HT29 colon adenocarcinoma cells. Brithish Journal of Pharmacology 150: 209-219.

Kozarski, M., A. Klaus, M. Niksic, D. Jakovljevic, J.P.F.G. Helsper, L.J.L.D. Van Griensven, 2011. Antioxidative and immunomodulating activities of polysaccharide extracts of the medicinal mushrooms Agaricus bisporus, Agaricus brasiliensis, Ganoderma lucidum and Phellinus linteus. Food Chemistry 129: 1667-1675.

Largent, D.L., 1973. How to identify mushrooms to genus I: Macroscopics Features. Mad River Press Inc. Eureka, California.

Largent, D.L., D. Johnson, R. Watling, 1977. How to identify mushrooms to genus III: Microscopics Features. Primera edición. Mad River Press Inc. Eureka, California.

Lee, I.H., R.L. Huang, C.T. Chen., H.C. Chen, W.C. Hsu, M.K. Lu, 2002. Antrodia camphorate polysaccharides exhibit antihepatitis B virus effects. FEMS Microbiology Letters 209: 61-65.

Maldonado, O., E.N. Jiménez, M.R.B. Guapillo, G.M. Ceballos, E. Méndez, 2010. Radicales libres y su papel en las enfermedades crónico-degenerativas. Revista Médica de la Universidad Veracruzana 10 (2): 32-39.

Mau, J.L., S.Y. Tsai, Y.H. Tseng, S.J. Huang, 2005a. Antioxidant properties of methanolic extracts from Ganoderma tsugae. Food Chemistry 93: 641-649.

Mau, J.L., S.Y. Tsai, Y.H. Tseng, S.J. Huang, 2005b. Antioxidant properties of hot water extracts from Ganoderma tsugae Murrill. LWT-Food Science and Technology 38: 589-597.

Nagaraj, K., N. Mallikarjun, R. Naika, T.M. Venugopal, 2014. Antioxidative activities of wild macro fungi Ganoderma applanatum (Pers.) Pat. Asian Journal of Pharmaceutical and Clinical Research 7: 166-171.

Penzete, G., A. Assunção, G.C. Dos Santos, A. Bracht, C. Giatti, C. Gandolfi, R. Marina, 2012. Antioxidant properties of the most common edible mushrooms consumed in Brazil. In: Andres S., 
Baumann N. (eds.), Musrooms: types, properties and nutrition. Nova Science Publishers Inc. New York. Pp. 285-295.

Pilotti, C.A., F.R. Sanderson, E. Aitken, W. Armstrong, 2004. Morphological variation and host range of two Ganoderma species from Papua New Guinea. Mycopathologia 158 (2): 151-265.

Postemsky, P., D. Figlas, S. Delmastro, R. Devalis, N. Curvetto, 2006. Optimizing Grifola sordulenta and Grifola gargal growth in agar and liquid nutrient media. Micología Aplicada International 18: 7-12.

Re, R., N. Pellegrini, A. Proteggente, A. Pannala, M. Yang, C. Rice Evans, 1999. Antioxidant activity applying an improved ABTS radical cation decolorization assay. Free Radical Biology and Medicine 26: 1231-1237.

Reis, F.S., A. Martins, L. Barros, I.C.F.R. Ferreira, 2012. Antioxidant properties and phenolic profile of the most widely appreciated cultivated mushrooms: a comparative study between in vivo and in vitro samples. Food and Chemical Toxicology 50: 1201-1207.

Ryvarden, L., 1991. Genera of polypores, nomenclature and taxonomy. Synopsis Fungorum 5. Fungiflora, Oslo.

Ryvarden, L., 2000. Studies in neotropical polypores 2: a preliminary key to neotropical species of Ganoderma with a laccate pileus. Mycologia 92: 181-191.

Ryvarden, L., R.L. Gilbertson, 1993. European Polypores, Part 1: Abortiporus-Lindtneria. Synopsis Fungorum 6. Fungiflora, Oslo.

Saltarelli, R., P. Ceccaroli, M. Lotti, A. Zambonelli, M. Buffalini, L. Casadei, L. Vallorani, V. Stocchi, 2009. Biochemical characterization and antioxidant activity of mycelium of Ganoderma lucidum from Central Italy. Food Chemistry 116: 143-151.

Sánchez, R, 1980. Macromicetos patógenos y destructores de la madera de los bosques de la Meseta Tarasca, Michoacán. Revista Ciencia Forestal 5: 4-19.

Shoji, O., 1999. Effect of water potencial on fruitbody formation of Lentinula edodes in sawdust-based substrate. Journal of Wood Science 45: 337-342.

Singdevsachan, S.K., J.K. Patra, K. Tayung, H. Thatoi, 2015. Chemical constituents, antioxidative and antibacterial properties of medicinal mushrooms collected from Similipal Biosphere Reserve, Odisha, India. Proceedings of the National Academy of Sciences, India Section B: Biological Sciences 87: 559-570.
Singleton, V.L., R. Orthofer, R.M. Lamuela-Raventos, 1999. Analysis of total phenols and other oxidation substrates and antioxidants by means of Folin-Ciocalteu reagent. Methods in Enzymology 299: 152-178.

Smina, T.P., J. Mathew, K.K. Janardhanan, T.P.A. Devasagayam, 2011. Antioxidant activity and toxicity profile of total triterpenes isolated from Ganoderma lucidum (Fr.) P. Karst occurring in South India. Environmental Toxicology and Pharmacology 32: 438446.

Stalpers, J.A., 1978. Identification of wood-inhabiting Aphyllophorales in pure culture. Studies in Mycology 16: 1-248.

Stamets, P., 2000. Growing gourmet and medicinal mushroom. $3^{\mathrm{a}}$. Edition. Ten Speed Pres, Olympia, WA. Berkeley, California.

Tamrakar, S., H.B. Tran, M. Nishida, S. Kaifuchi, H. Suhara, K. Doi, K. Fukami, G.P. Parajuli, K. Shimizu, 2016. Antioxidative activities of 62 wild mushrooms from Nepal and the phenolic profile of some selected species. Journal of Natural Medicine 70 (4): 769-779.

Torres-Torres, M.G., L. Ryvarden, L. Guzmán-Dávalos, 2015. Ganoderma subgénero Ganoderma en México. Revista Mexicana de Micología 41: 27-45.

Tseng, Y.H., J.H. Yang, J.L. Mau, 2008. Antioxidant properties of polysaccharides from Ganoderma tsugae. Food Chemistry 107 (2): 732-738.

Wasser, S.P., A.L. Weis, 1997. Medicinal mushrooms. In: Nevo, E. (ed.), Reishi mushroom (Ganoderma lucidum, (Curtis: Fr.), P. Karst), Peledfus Public House, Haifa. Pp. 1-39.

Yang, J.H., Y.M. Du, R.H. Huang, L.P. Sun, H. Liu, X.H. Gao, 2005. Chemical modification and antitumor activity of Chinese lacquer polysaccharide from lac tree Rhus vemicifera. Carbohydrate Polymers 59: 101-107.

Yoon, S. J., M.A. Yu, Y.R. Pyun, J.K. Hwang, D.C. Chu, L.R. Juneja, 2003. The nontoxic mushroom Auricularia auricula contains a polysaccharides with anticoagulant activity mediated by antithrombin. Thrombosis Research 112: 151-158.

Yoshikawa, T., S. Toyokuni, Y. Yamamoto, Y. Naito, 2000. Free Radicals in Chemistry Biology and Medicine. OICA International, Londres. 